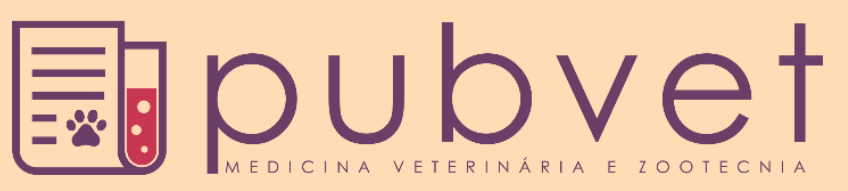

https://doi.org/10.31533/pubvet.v15n09a925.1-10

\title{
Pancreatite crônica esclerosante em cão: Relato de caso
}

\author{
Gisele Augusta Amorim de $\operatorname{Lemos}^{1 *}{ }^{\bullet}$, Felipe Martins Negreiros Navolar ${ }^{1} \bullet \mathbb{D}$, Ana Paula \\ Frederico Rodrigues Loureiro Bracarense ${ }^{2} \theta$ (D)
}

${ }^{1}$ Médicos Veterinários residentes da Universidade Estadual de Londrina (UEL), Departamento de Medicina Veterinária Preventiva, Laboratório de Patologia Animal, Londrina - PR

${ }^{2}$ Professora Associada da UEL, Departamento de Medicina Veterinária Preventiva, Laboratório de Patologia Animal, Londrina - PR Brasil. *Autor para correspondência, e-mail: anapaula@uel.br

Resumo. Este trabalho tem como objetivo relatar um caso de fibrose pancreática esclerosante em um animal com diabetes mellitus, descrevendo os aspectos clínicos e anatomopatológicos envolvidos na doença em uma cadela, poodle, nove anos, atendida em um Hospital-Escola Veterinário. O animal apresentava picos de hipoglicemia e hiperglicemia, sem resposta ao tratamento insulínico e prognóstico desfavorável. No exame de autópsia, os principais achados anatomopatológicos foram atrofia e fibrose pancreática esclerosante, cistite supurativa e enfisematosa e degeneração hepatocelular gordurosa e glicogênica. Com o auxílio de métodos histoquímicos foi possível identificar a deposição/substituição do interstício pancreático por tecido fibroso.

Palavras-chave: Cistite enfisematosa, diabetes mellitus, esteatose, fibrose pancreática, pancreatite crônica, pâncreas

\section{Chronic sclerosing pancreatitis in a dog: Case report}

Abstract. This study aims to report a case of pancreatic fibrosis describing the clinical and anatomopathological aspects involved in the disease in a 9-year-old female poodle. The animal was attended at a Veterinary Hospital showing peaks of hypoglycemia and hyperglycemia, with no response to insulin treatment, and poor prognosis. In the autopsy exam, the main findings were pancreatic atrophy and fibrosis, suppurative and emphysematous cystitis, steatosis and hepatocellular glycogenic degeneration. By using histochemical methods, it was possible to identify the deposition/replacement of the pancreatic interstitium by fibrotic tissue.

Keywords: Emphysematous cystitis, glucogenic degeneration, diabetes mellitus, chronic pancreatitis, pancreas

\section{Pancreatitis esclerosante crónica en un perro: Reporte de un caso}

Resumen. Este estudio tiene como objetivo reportar un caso de fibrosis pancreática describiendo los aspectos clínicos y anatomopatológicos involucrados en la enfermedad en hembra Poodle de 9 años, atendida en un Hospital-Universitario Veterinario. El animal presentaba picos de hipoglucemia e hiperglucemia, sin respuesta al tratamiento con insulina y pronóstico desfavorable. En el examen de autopsia, los principales hallazgos fueron atrofia y fibrosis pancreática, cistitis supurativa y enfisematosa, esteatosis y degeneración glucogénica hepatocelular. Con la ayuda de métodos histoquímicos, fue posible identificar el depósito / reemplazo del intersticio pancreático por tejido fibrótico.

Palabras clave: Cistitis enfisematosa, degeneración glucogénica, diabetes mellitus, pancreatitis crónica, páncreas 


\section{Introdução}

O pâncreas é um órgão que desempenha importantes funções no organismo dos seres vivos, inclusive nos pequenos animais. Esta glândula é composta por células com funções endócrinas e exócrinas, que resultam na produção de hormônios (insulina e glucagon), os quais controlam os níveis de glicose no sangue, e ainda, de enzimas que ajudam no processo digestivo e facilitam a absorção dos nutrientes (Berman et al., 2020; Valenciano \& Cowell, 2019).

A pancreatite é uma doença inflamatória que atinge o pâncreas e é considerada a desordem mais comum do pâncreas exócrino dos pequenos animais. Esta entidade pode ser classificada em pancreatite crônica e pancreatite aguda (Jericó et al., 2015). Os animais mais afetados são cães de meia idade a idosos e castrados, sem predileção sexual (Nelson \& Couto, 2015). Foram relatadas em algumas raças predisposição racial como em cocker spaniel inglês (Birchard \& Sherding, 2008; Watson et al., 2011), cavalier king charles spaniel, collie, boxer (Watson et al., 2007; Wiberg, 2004), schnauzer miniatura, poodle (Birchard \& Sherding, 2008) e raças terrier (Nelson \& Couto, 2015). Os animais com pancreatite aguda apresentam lesões reversíveis como infiltrado neutrofílico e focos de necrose que, obtendo o diagnóstico e tratamento corretos, apresentam resolução favorável. Entretanto, se as lesões ao tecido pancreático forem graves, não forem tratadas corretamente ou forem recorrentes, os animais podem apresentar complicações clínicas, desenvolver lesões pancreáticas irreversíveis (Nelson \& Couto, 2015), dentre as quais a pancreatite crônica (Xenoulis \& Steiner, 2010) e ir a óbito. A pancreatite crônica é uma alteração frequente, mas subdiagnosticada em cães (Watson et al., 2007), sendo considerada uma doença reemergente nos caninos domésticos (Watson, 2012). É definida como uma doença inflamatória contínua do pâncreas e caracterizada por alterações morfológicas e funcionais irreversíveis como fibrose e atrofia do parênquima pancreático (Xenoulis \& Steiner, 2010), que podem cursar com dor abdominal, insuficiência pancreática exócrina (IPE) e diabetes mellitus (DM) (Steiner, 2008). Infere-se que o desenvolvimento de IPE ou DM ocorre apenas quando há destruição de mais de três quartos da reserva funcional pancreática (Marcato, 2010; Ramsey et al., 2017).

Exames laboratoriais para o diagnóstico da pancreatite aguda e crônica até o presente momento demonstram-se poucos sensíveis e específicos (Newman et al., 2004; Steiner, 2003). Para estabelecer o diagnóstico deve-se realizar a avaliação cuidadosa da história do animal, seguida de exame físico e exames laboratoriais. Entretanto, hemograma, exames bioquímicos, avaliação da atividade da amilase e lipase, imunorreatividade da tripsina (Mansfield, 2020), mensuração da lipase sérica (Simpson et al., 1991) apresentam baixa especificidade e são considerados pouco úteis no diagnóstico da doença. Atualmente, o teste clínico-patológico mais utilizado para o diagnóstico de pancreatite é a lipase pancreática canina (LPC), um snap test tipo Elisa que detecta a enzima LPC que é sintetizada especificamente no pâncreas e apresenta poucos fatores de interferência externos no resultado. É excelente para o diagnóstico da pancreatite aguda, mas não apresenta bons resultados nos casos de pancreatite crônica (Mansfield, 2020).

Os exames de imagem que podem auxiliar no diagnóstico e prognóstico da pancreatite são a ultrassonografia (Kealy et al., 2012; Ruaux, 2003; Xenoulis \& Steiner, 2010), radiografia (Steiner, 2003), tomografia computadorizada (Ruaux, 2003; Xenoulis \& Steiner, 2010). Todas têm seus prós e contras, e devem ser bem pensadas para serem aplicadas na conduta clínica.

Atualmente não existe tratamento para reverter ou retardar a pancreatite crônica (Ramsey et al., 2017). Dentro deste contexto a pancreatite crônica, por não possuir um diagnóstico clínico e laboratorial simples, tem como método mais confiável para o diagnóstico definitivo desta condição em cães, o exame histopatológico (Steiner, 2003).

A DM é uma síndrome metabólica caracterizada por hiperglicemia, e é uma endocrinopatia muito comum no cotidiano da rotina das clínicas veterinárias, diagnosticada principalmente em cães e gatos (Martin \& Capen, 1985; Zachary et al., 2012). Independente da etiologia, os cães e gatos com DM apresentam hiperglicemia e glicosúria, resultando em sinais clínicos como poliúria, polidipsia, polifagia e perda de peso (Rucinsky et al., 2010; Zachary et al., 2012). 
A pancreatite crônica, por outro lado, é listada como uma das etiologias da DM e da IPE, uma vez que causa o comprometimento progressivo da função endócrina e exócrina, podendo chegar a perda total do parênquima pancreático no estágio final da doença (Martin \& Capen, 1985; Zachary et al., 2012). A pancreatite crônica causa perda de função permanente no órgão, além de haver perda contínua do parênquima, o qual é substituído por tecido fibroso. Pode se desenvolver silenciosamente até que haja a perda de $80 \%$ a $90 \%$ do tecido funcional pancreático (Watson, 2015).

O presente relato objetivou descrever os achados clínicos, laboratoriais e anatomopatológicos em uma cadela com fibrose pancreática esclerosante e com diabetes mellitus.

\section{Relato de caso}

Trata-se de uma cadela, poodle, de nove anos de idade, de 5,6 kg, que foi encaminhada para o Hospital Veterinário da Universidade Estadual de Londrina, com queixa de apatia e anorexia, decúbito obrigatório, tremor muscular e alheia ao meio. $\mathrm{O}$ animal recebia alimentação caseira, o protocolo vacinal e a vermifugação estavam desatualizados.

Ao exame clínico geral, as mucosas estavam rosadas, a temperatura corporal normal, frequência cardíaca e respiratória normais, nível de consciência deprimido, pulso arterial regular, estado nutricional magro, decúbito lateral direito obrigatório, olhos com discreta secreção aquosa bilateral e opacidade de cristalino bilateral, alopecia simétrica bilateral, dor à palpação abdominal, hepatomegalia, esplenomegalia, e resposta neurológica reduzida.

Foram solicitados exames bioquímicos, hematológicos, hemogasometria e urinálise. Das enzimas bioquímicas solicitadas a fosfatase alcalina (FA) apresentou $876 \mathrm{U} / \mathrm{L}$, e a alanina aminotransferase (ALT) 64 U/L. No hemograma observou-se anemia regenerativa, linfopenia e trombocitopenia. Na aferição da glicemia constatou-se $584 \mathrm{mg} / \mathrm{dL}$, caracterizando hiperglicemia. O exame de urinálise revelou proteinúria discreta, glicosúria moderada e bacteriúria acentuada. $\mathrm{O}$ exame de hemogasometria revelou acidose metabólica sem acidemia, por compensação respiratória, além de hipocalemia, hipercalcemia e hiperlactatemia.

O animal foi internado para monitoramento do estado clínico e do índice glicêmico, e apresentou vários picos de hipoglicemia e hiperglicemia. No entanto, a evolução do quadro clínico foi desfavorável, e optou-se pela submissão do animal à eutanásia. No exame de autópsia realizado no Laboratório de Patologia Animal (LAP) da Universidade Estadual de Londrina, o animal exibia condição corporal magra, e áreas multifocais a coalescentes de rarefação pilosa, predominantemente nas regiões de flanco e lombar.

Macroscopicamente o pâncreas estava diminuído de tamanho e endurecido. Ao exame microscópico (Figura 1), demonstrou abundante deposição de tecido conjuntivo fibroso circunscrevendo os lóbulos e ductos pancreáticos. Havia ácinos atróficos nos fragmentos analisados e não foram evidenciadas ilhotas de Langerhans. Infiltrando a região intersticial e fibrótica, havia moderado infiltrado inflamatório linfohistiocítico, caracterizando uma pancreatite crônica esclerosante multifocal a coalescente acentuada.

A avaliação macroscópica do fígado constatou hepatomegalia difusa, com áreas de evidenciação do padrão lobular e aumento da friabilidade hepática, caracterizando uma degeneração hepática difusa. Microscopicamente (Figura 2) no fígado havia esteatose hepática macrovesicular e microvesicular multifocal acentuada, difusa, degeneração glicogênica focalmente extensa moderada e hepatite linfoplasmocitária multifocal moderada.

Adicionalmente, discretas alterações renais foram observadas como necrose tubular aguda e áreas de infarto renal crônico, caracterizadas pela fibrose. O interior da bexiga urinária continha áreas multifocais a coalescentes hemorrágicas além de acúmulo de exudato supurativo fétido e sua parede exibia espessamento difuso acompanhado de crepitação pela presença de gás. A histopatologia destacou a formação de cavitações sem conteúdo na submucosa, onde havia presença de gás, caracterizando uma 
cistite supurativa e enfisematosa difusa acentuada (Figura 3). Nos demais órgãos, alterações significativas não foram visualizadas.
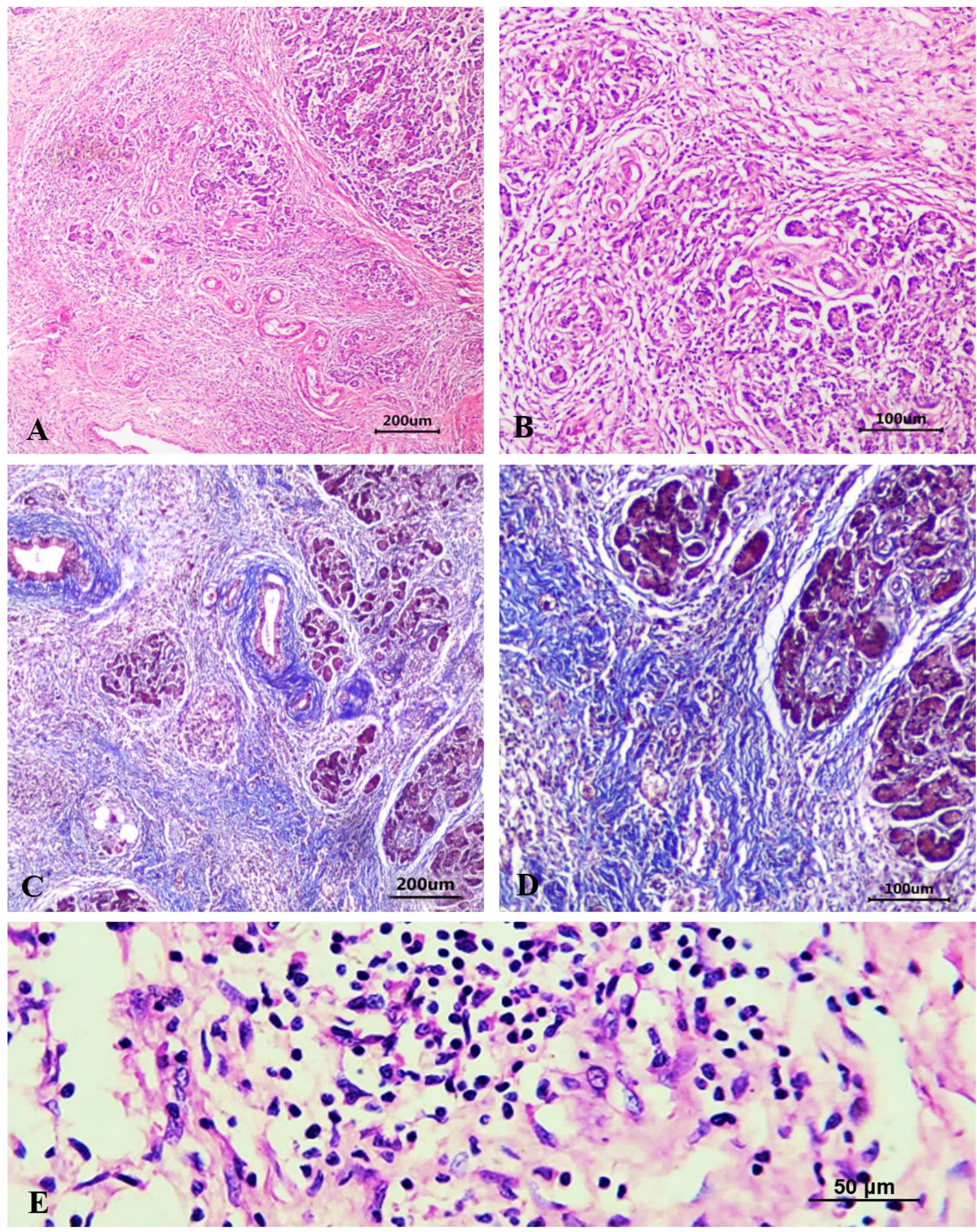

Figura 1. Aspectos histopatológicos e histoquímicos da pancreatite crônica esclerosante de um cão. A. Corte de pâncreas mostrando intensa fibrose inter- e intralobular, com grande redução do parênquima exócrino [HE; barra= $200 \mu \mathrm{m}$ ]. B. Em alguns lóbulos, os ácinos estão reduzidos em número e volume, sem visualização de ilhotas de Langerhans $[\mathrm{HE}$; barra $=100 \mu \mathrm{m}]$. C.Marcação intensa de tecido conjuntivo fibroso (azul), aos arredores de ácinos pancreáticos atróficos [tricrômico de Masson; barra $=200 \mu \mathrm{m}$ ]. D. Evidenciação dos ácinos atróficos [tricrômico de Masson; barra $=100 \mu \mathrm{m}$. E. Em meio à fibrose, observa-se acentuado infiltrado inflamatório crônico linfohistiocítico [HE; barra $=50 \mu \mathrm{m}]$. 

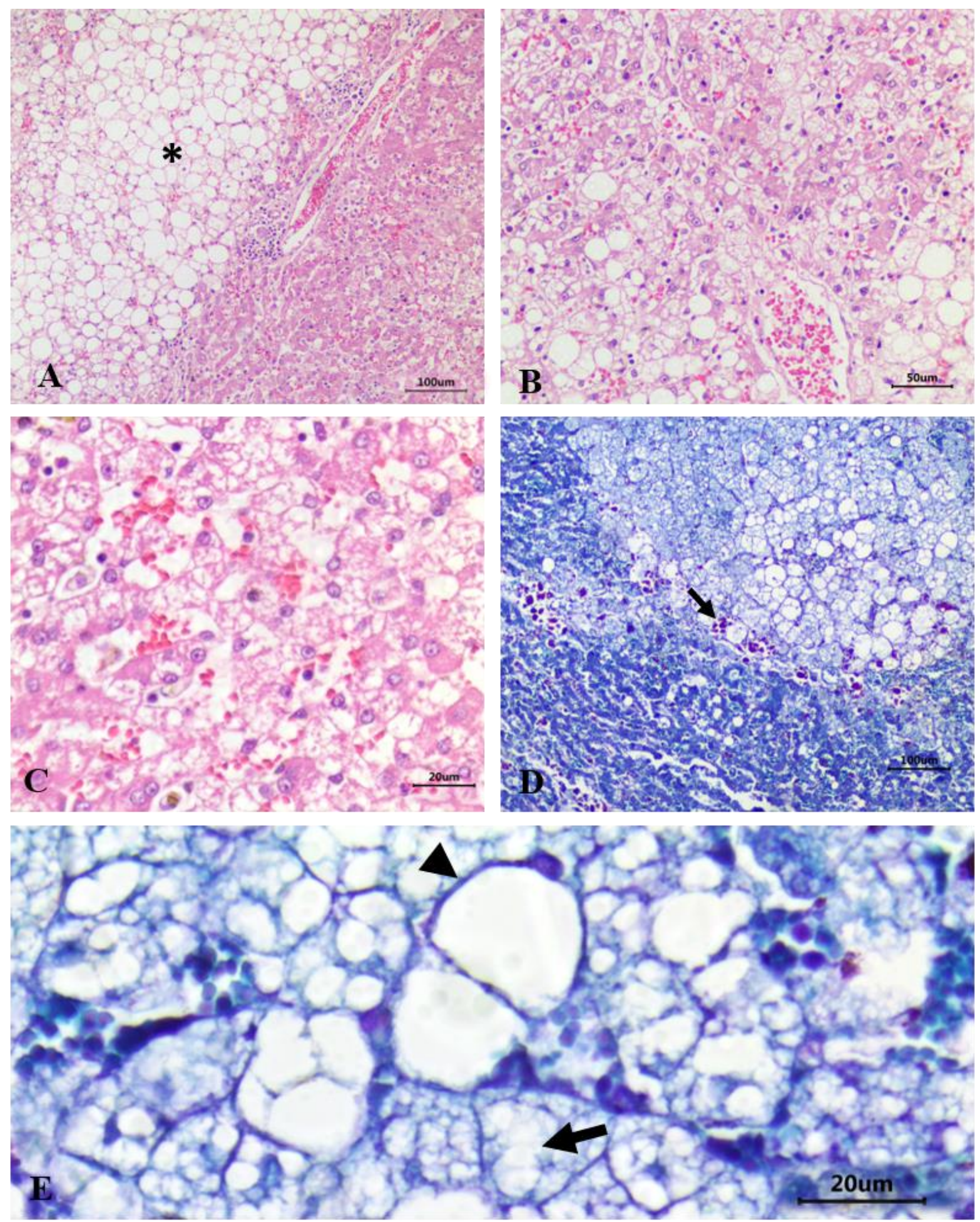

Figura 2. Aspectos histopatológicos e histoquímicos da lipidose hepática e degeneração glicogênica em um fígado de cão. A. Do lado direito os hepatócitos exibem grandes vacúolos intracitoplasmáticos, com deslocamento nuclear para a periferia celular $(*)$, em contraste com o lado direito, visualiza-se o parênquima hepático normal, [HE; barra $=100$ $\mu \mathrm{m}]$. B. Lipidose em maior aumento demonstrando apresentação microvacuolar e macrovacuolar dos hepatócitos, $[\mathrm{HE} ;$ barra $=50 \mu \mathrm{m}]$. C. Parênquima hepático que demonstra células em aspecto vegetal, com vacuolização indistinta intracitoplasmáticas e formação de traves eosinofílicas e sem deslocamento nuclear, [HE; barra = 20 $\mu \mathrm{m}$ ]. D. Marcação positiva nas células de Kupffer e negativa para os hepatócitos, [ácido periódico de Schiff (PAS); barra $=100 \mu \mathrm{m}]$. E. Hepatócitos com macrovacúolo e núcleo excêntrico (cabeça da seta) e hepatócitos com microvacúolos intracitoplasmáticos (seta), [PAS; barra $=20 \mu \mathrm{m}]$. 

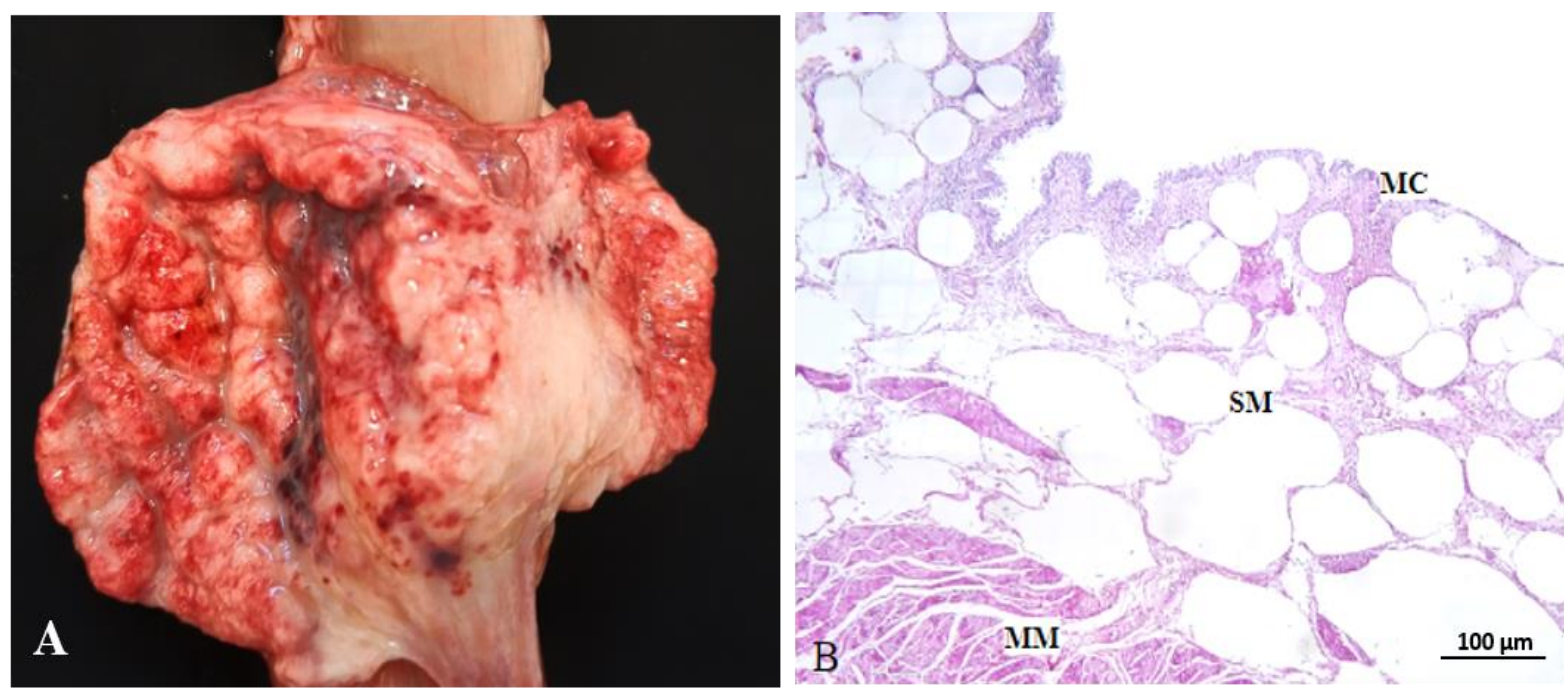

Figura 3. A. Cistite enfisematosa na bexiga de cão. B. Fotomicrografia de fragmento histológico demonstrando a preservação do urotélio da mucosa (MC) e da camada muscular (MM), enquanto na camada submucosa (SM) está expandida pela presença de múltiplas vesículas bem delimitadas formadas pelo acúmulo de gás resultante da fermentação bacteriana [HE; bar $=100 \mu \mathrm{m}]$.

\section{Discussão}

Dentre os principais achados deste caso destacam-se a esclerose, inflamação crônica e atrofia pancreática exócrina severas que reiteram as alterações anatomopatológicas de uma pancreatite crônica em seu estágio final. Com isso, traz-se a oportunidade de discutir sobre as divergentes classificações que apontam a histopatologia do pâncreas a fim do diagnóstico de pancreatite na veterinária e a relação desta doença com a diabetes mellitus.

Estudos apontam que a prevalência da pancreatite aguda e crônica ainda é desconhecida, em vista que a ocorrência de casos é maior do que o diagnóstico em si (Xenoulis et al., 2008). Informações publicadas sobre os achados clínicos, anatomopatológicos e histopatológicos de cães com pancreatite crônica, bem como quaisquer associações entre eles, são muito limitadas (Watson et al., 2010a; Watson et al., 2011).

Os dados epidemiológicos sobre pancreatite crônica são quase que exclusivamente baseados em estudos retrospectivos e post mortem. Destes resultados obtidos, a pancreatite crônica estava presente em um número inesperadamente grande nos caninos do estudo, variando entre $34 \%$ e $64 \%$ de todos os casos examinados (Newmann et al., 2004, Watson et al., 2007). No entanto, esses estudos não fornecem informações sobre como os achados histopatológicos se traduzem em doença clínica; portanto, a importância clínica desses achados permanece desconhecida. Embora faltem estudos clínicos sobre a incidência de pancreatite crônica canina, a experiência clínica sugere que a condição é diagnosticada com muito menos frequência na prática clínica, o que pode sugerir que muitos casos de pancreatite crônica são subdiagnosticados ou permanecem subclínicos (Xenoulis et al., 2008).

O diagnóstico clínico de pancreatite crônica é difícil e desafiador, uma vez que a doença geralmente é subclínica ou está associada a sinais clínicos inespecíficos leves e anormalidades laboratoriais. Desta forma, a maioria dos casos de pancreatite crônica canina e felina permanecem sem diagnóstico definitivo (Steiner, 2003; Newmann et al.,2004).

Os sinais clínicos da pancreatite podem variar conforme a gravidade da doença (Watson, 2015;

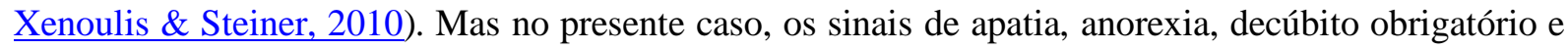
neurológicos, opacidade do cristalino, aumento de enzimas hepáticas como FA e ALT e a acentuada glicosúria, e principalmente o alto índice glicêmico (Marco et al., 1999) fizeram ponderar o diagnóstico de diabetes mellitus. Aumentos de colesterol nesta endocrinopatia também são citados, mas no presente caso, não foi mensurado (Berman et al., 2020). 
Quanto à classificação histológica entre a pancreatite aguda e crônica ainda existem muitas divergências. Na veterinária ainda não existe um sistema de diagnóstico e classificação de pancreatite padronizado assim como na medicina humana. Atualmente, a classificação na veterinária é adaptada para a realidade dos cães e gatos com base na padronização humana (Newman et al., 2004; Reilly, 2006). Na veterinária, a diferenciação se dá a partir do infiltrado inflamatório existente no tecido pancreático, em que a aguda é caracterizada pela presença de infiltrado neutrofílico, e a crônica pelo predomínio de linfócitos, classificada como crônica. Entretanto, não somente o infiltrado deve ser considerado para garantir o acerto desta classificação, mas também, utilizar outros achados histopatológicos como a necrose tecidual na sua forma aguda, e a fibrose na sua forma crônica ajudam a fechar o diagnóstico mais corretamente (Xenoulis et al., 2008).

O diagnóstico de pancreatite crônica no presente caso foi confirmado após o exame de autópsia, onde o exame histopatológico revelou severas alterações irreversíveis, que compreendiam até $80 \%$ de comprometimento do tecido pancreático. Destas alterações, a fibrose, atrofia acinar e a inflamação crônica estavam presentes (Xenoulis, 2015; Xenoulis et al., 2008). A destruição de grande parte do parênquima pancreático não é incomum em cães que já sofreram com quadros de pancreatite aguda, mesmo que em episódios subclínicos (Watson, 2012). Em alguns casos, à microscopia é possível notar lesões nas células das ilhotas pancreáticas. Em contrapartida, por ser uma desordem funcional, não necessariamente é acompanhada de lesões morfologicamente visíveis. Nesses casos, contanto que o paciente se recupere, há o retorno histológico e funcional do pâncreas, enquanto que na pancreatite crônica, há mudança histológica permanente e perda progressiva da função exócrina e endócrina, que pode progredir para IPE e/ou DM (Watson, 2003). Câmara et al. (2018) relatam um caso de destruição pancreática total causada por pancreatite em um cão, e este também apresentava sinais de diabetes mellitus. No presente caso, nos cortes analisados, não foram notadas a presença das ilhotas de Langerhans. Considerando este achado, deduzimos que houve a substituição do parênquima pancreático endócrino pela fibrose, e consequentemente levou a uma hipofunção das células das ilhotas pancreáticas, resultando no quadro de DM.

Um estudo utilizando camundongos no intuito de estudar a relação entre a diabetes e a pancreatite crônica demonstrou que a diabetes agravou a resposta inflamatória, intensificou a atrofia e morte das células acinares e estimulou a fibrose pancreática (Zechner et al., 2014). Contudo, este estudo provoca uma controvérsia em relação a etiologia da DM em decorrência da perda do tecido pancreático devido à pancreatite crônica. Sobre as lesões hepáticas encontradas neste caso, a prevalência destas lesões em cães com pancreatite crônica não se revelou significativa (Watson et al., 2010b). Os achados de degeneração hepática encontrados foram associados à DM, uma vez que esta endocrinopatia causa o aumento da mobilização de gordura, resultando em acúmulo de gordura nos hepatócitos e aumento do volume do órgão, macroscopicamente visível pela hepatomegalia, palidez e aumento da friabilidade hepática (Rucinsky et al., 2010).

Na DM a hiperglicemia é resultado de uma deficiência relativa ou absoluta da sintetização e secreção de insulina pelas células das ilhotas $\beta$ (DM tipo 1), ou de uma falha das células-alvo em reconhecer e responder à insulina (DM tipo 2) (Gilor et al., 2016). Nos caninos, a DM é predominantemente do tipo 1 , ao contrário dos felinos e humanos (Nelson \& Reusch, 2014). Sabe-se que a DM tipo 1 pode ser primária (autoimune) ou secundária à lesão pancreática extensa, como no presente caso. Estudos em humanos associam a esteatose hepática como alteração secundária a DM tipo 2, enquanto a DM tipo 1 gera principalmente hepatopatia glicogênica (Messeri et al., 2012). O mecanismo de desenvolvimento da hepatopatia glicogênica nos humanos ainda não é totalmente elucidado. Não obstante, sabe-se que a partir do controle glicêmico do paciente, há a reversão do quadro de degeneração glicogênica hepática (Hudacko et al., 2008).

No presente caso, o animal apresentava uma cistite enfisematosa marcante, justificada pela endocrinopatia da DM. É comum que a infecção do trato urinário inferior se torne recorrente, e está geralmente associada a microrganismos fermentadores de glicose como Proteus sp., Aerobacter aerogenes e Escherichia coli, os quais induzem a formação de gás na parede e lúmen da bexiga, tornando a cistite enfisematosa uma lesão característica em animais com essa endocrinopatia (Gregory, 2003; Maxie, 2007; Moon et al., 2014). 


\section{Considerações finais}

O presente relato ilustrou lesões clínico-patológicas características da pancreatite crônica canina que são pouco destacadas na literatura veterinária. Embora essa seja uma doença subdiagnosticada na rotina clínica e sua real prevalência ainda seja desconhecida, a demonstração dos achados clínicos, laboratoriais e anatomopatológicos forneceu dados relevantes para a melhor compreensão das características dessa enfermidade e, também, reiterou a necessidade crescente da realização de estudos prospectivos e novas pesquisas para o estabelecimento de métodos diagnósticos mais efetivos.

\section{Referências bibliográficas}

Berman, C. F., Lindquist, E., \& Lobetti, R. G. (2020). Comparison of clinical findings in 293 dogs with suspect acute pancreatitis: Different clinical presentation with left lobe, right lobe or diffuse involvement of the pancreas. Journal of the South African Veterinary Association, 91(1), 1-10. DOI: https://doi.org/10.4102/jsava.v91i0.2022

Birchard, S. J., \& Sherding, R. G. (2008). Manual Saunders: clínica de pequenos animais. In Ed. Roca (Vol. 3).

Câmara, B. O. S., Viana, F. A. B., Ribeiro, B. N. T., Ocarino, N. M., Nepomuceno, A. C., \& Serakides, R. (2018). Um caso raro de destruição total do pâncreas por pancreatite em cão. Arquivo Brasileiro de Medicina Veterinária e Zootecnia, 70(5), 1655-1659. DOI https://doi.org/10.1590/1678-4162$\underline{10103}$

Gilor, C., Niessen, S. J. M., Furrow, E., \& DiBartola, S. P. (2016). What's in a name? Classification of diabetes mellitus in veterinary medicine and why it matters. Journal of Veterinary Internal Medicine, 30(4), 927-940. DOI: https://doi.org/10.1111/jvim.14357

Gregory, C. R. (2003). Urinary system. In K. S. Latimer, E. A. Mahaffey, \& K. W. Prasse (Eds.), Duncan \&Prasse's Veterinary laboratory medicine: clinical patology (pp. 231-257). Blackwell Publishing Company.

Hudacko, R. M., Manoukian, A. V, Schneider, S. H., \& Fyfe, B. (2008). Clinical resolution of glycogenic hepatopathy following improved glycemic control. Journal of Diabetes and Its Complications, 22(5), 329-330. DOI: https://doi.org/10.1016/j.jdiacomp.2007.11.004

Jericó, M. M., Kogika, M. M., \& Andrade Neto, J. P. (2015). Tratado de medicina interna de cães e gatos. Guanabara Koogan.

Kealy, J. K., McAllister, H., \& Graham, J. P. (2012). Radiologia e ultrassonografia do cão e do gato (Vol. 1). Elselvier Saunders.

Mansfield, C. (2020). Pancreatitis in dog. In D. Bruyette (Ed.), Clinical small animal internal medicine. John Wiley \& Sons.

Marcato, J. A. (2010). Pancreatite em cães.

Marco, V., Amaral, R. C., Jericó, M. M., Silva, R. D., \& Simões, D. M. (1999). Diagnóstico de diabetes mellitus na espécie canina e avaliação a longo prazo da terapia insulínica através das concentrações séricas de hemoglobina glicosilada. Revista de Educação Continuada Em Medicina Veterinária e Zootecnia Do CRMV-SP, 2(2), 23-28. DOI: https://doi.org/10.36440/recmvz.v2i2.3382

Martin, S. L., \& Capen, C. C. (1985). The endocrine system. In P. W. Pratt (Ed.), Feline medicine. American Veterinary.

Maxie, M. G. (2007). The urinary system. In K. Jubb \& N. C. Palmer (Eds.), Pathology of domestic animals (pp. 425-522). Saunders Elsevier.

Messeri, S., Messerini, L., Vizzutti, F., Laffi, G., \& Marra, F. (2012). Glycogenic hepatopathy associated with type 1 diabetes mellitus as a cause of recurrent liver damage. Annals of Hepatology, 11(4), 554558. DOI: https://doi.org/10.1016/s1665-2681(19)31472-3

Moon, R., Biller, D. S., \& Smee, N. M. (2014). Emphysematous cystitis and pyelonephritis in a nondiabetic dog and a diabetic cat. Journal of the American Animal Hospital Association, 50(2), 124-129. DOI: https://doi.org/10.5326/jaaha-ms-5972

Nelson, R W, \& Couto, C. G. (2015). Medicina interna de pequenos animais (Issue 1). Elsevier Editora. 
Nelson, Richard W, \& Reusch, C. E. (2014). Animal models of disease: classification and etiology of diabetes in dogs and cats. Journal of Endocrinology, 222(3), T1-T9. DOI: https://doi.org/10.1530/joe-14-0202

Newman, S., Steiner, J., Woosley, K., Barton, L., Ruaux, C., \& Williams, D. (2004). Localization of pancreatic inflammation and necrosis in dogs. Journal of Veterinary Internal Medicine, 18(4), 488493. DOI: https://doi.org/10.1111/j.1939-1676.2004.tb02572.x

Ramsey, M. L., Conwell, D. L., \& Hart, P. A. (2017). Complications of chronic pancreatitis. Digestive Diseases and Sciences, 62(7), 1745-1750. DOI: 10.1007/s10620-017-4518-X

Reilly, C. (2006). BSAVA Manual of canine and feline gastroenterology. Journal of Small Animal Practice, 47(4), 228.

Ruaux, C. G. (2003). Diagnostic approaches to acute pancreatitis. Clinical Techniques in Small Animal Practice, 18(4), 245-249. DOI: https://doi.org/10.1016/s1096-2867(03)00072-0

Rucinsky, R., Cook, A., Haley, S., Nelson, R., Zoran, D. L., \& Poundstone, M. (2010). AAHA diabetes management guidelines for dogs and cats. Journal of the American Animal Hospital Association, 46(3), 215-224. DOI: https://doi.org/10.5326/0460215

Simpson, K. W., Simpson, J. W., Lake, S., Morton, D. B., \& Batt, R. M. (1991). Effect of pancreatectomy on plasma activities of amylase, isoamylase, lipase and trypsin-like immunoreactivity in dogs. Research in Veterinary Science, 51(1), 78-82. DOI: https://doi.org/10.1016/0034-5288(91)90035-m

Steiner, J. M. (2003). Diagnosis of pancreatitis. Veterinary Clinics: Small Animal Practice, 33(5), 11811195. DOI: 10.1016/s0195-5616(03)00061-5

Steiner, J. M. (2008). Exocrine pancreas. Small Animal Gastroenterology, 283-306.

Valenciano, A. C., \& Cowell, R. L. (2019). Cowell and Tyler's diagnostic cytology and hematology of the dog and cat-E-Book. Elsevier Health Sciences. DOI: https://doi.org/10.1016/c2016-0-02017-x

Watson, P. (2003). Exocrine pancreatic insufficiency as an end stage of pancreatitis in four dogs. The Journal of small animal practice. 44. 306-12. http://dx.doi.org/10.1111/j.1748-5827.2003.tb00159.x.

Watson, P. J., Archer, J., Roulois, A. J., Scase, T. J., \& Herrtage, M. E. (2010a). Observational study of 14 cases of chronic pancreatitis in dogs. Veterinary Record, 167(25), 968-976. DOI: https://doi.org/10.1136/vr.c4912

Watson, P. J., Roulois, A. J. A., Scase, T. J., Irvine, R., \& Herrtage, M. E. (2010b). Prevalence of hepatic lesions at post-mortem examination in dogs and association with pancreatitis. Journal of Small Animal Practice, 51(11), 566-572. DOI: https://doi.org/10.1111/j.1748-5827.2010.00996.x

Watson, P. J., Roulois, A. J. A., Scase, T., Johnston, P. E. J., Thompson, H., \& Herrtage, M. E. (2007). Prevalence and breed distribution of chronic pancreatitis at post-mortem examination in first-opinion dogs. Journal of Small Animal Practice, 48(11), 609-618. DOI: https://doi.org/10.1111/j.1748$\underline{\text { 5827.2007.00448.x }}$

Watson, P. J., Roulois, A., Scase, T., Holloway, A., \& Herrtage, M. E. (2011). Characterization of chronic pancreatitis in English Cocker Spaniels. Journal of Veterinary Internal Medicine, 25(4), 797-804. DOI: https://doi.org/10.1111/j.1939-1676.2011.0744.x

Watson, P. (2015). Pancreatitis in dogs and cats: definitions and pathophysiology. Journal of Small Animal Practice, 56(1), 3-12. DOI: https://doi.org/10.1111/jsap.12293

Watson, P. (2012). Chronic pancreatitis in dogs. Topics in Companion Animal Medicine, 27(3), 133139. https://doi.org/10.1053/j.tcam.2012.04.006.

Wiberg, M. E. (2004). Pancreatic acinar atrophy in German shepherd dogs and rough-coated Collies. Etiopathogenesis, diagnosis and treatment. A review. Veterinary Quarterly, 26(2), 61-75. DOI: https://doi.org/10.1080/01652176.2004.9695169

Xenoulis, P G. (2015). Diagnosis of pancreatitis in dogs and cats. Journal of Small Animal Practice, 56(1), 13-26. DOI: https://doi.org/10.1111/jsap.12274

Xenoulis, P G, \& Steiner, J. M. (2010). Lipid metabolism and hyperlipidemia in dogs. The Veterinary Journal, 183(1), 12-21. DOI: https://doi.org/10.1016/j.tvj1.2008.10.011 
Xenoulis, Panagiotis G, Suchodolski, J. S., \& Steiner, J. M. (2008). Chronic pancreatitis in dogs and cats. Compendium, 30(3), 166-181.

Zachary, J. F., McGavin, D., \& McGavin, M. D. (2012). Bases da patologia em veterinária. Elsevier Brasil.

Zechner, D., Knapp, N., Bobrowski, A., Radecke, T., Genz, B., \& Vollmar, B. (2014). Diabetes increases pancreatic fibrosis during chronic inflammation. Experimental Biology and Medicine, 239(6), 670-676. DOI: https://doi.org/10.1177/1535370214527890

Histórico do artigo:

Recebido: 28 de abril de 2021 .

Aprovado: 15 de junho de 2021.
Licenciamento: Este artigo é publicado na modalidade Acesso Aberto sob a licença Creative Commons Atribuição 4.0 (CC-BY 4.0), a qual permite uso irrestrito, distribuição, reprodução em qualquer meio, desde que $\mathrm{o}$ autor e a fonte sejam devidamente creditados. 